\title{
Direct measurement of bending stiffness and estimation of Young's modulus of vertically aligned carbon nanofibers
}

\author{
F. A. Ghavanini, ${ }^{1, a)}$ H. Jackman, ${ }^{2}$ P. Lundgren, ${ }^{1}$ K. Svensson, ${ }^{2}$ and P. Enoksson ${ }^{1}$ \\ ${ }^{1}$ Department of Microtechnology and Nanoscience, Chalmers University of Technology, 41296 Göteborg, \\ Sweden \\ ${ }^{2}$ Department of Physics and Electrical Engineering, Karlstad University, SE-651 88 Karlstad, Sweden
}

(Received 14 January 2013; accepted 16 April 2013; published online 20 May 2013)

\begin{abstract}
The bending stiffness of individual, as-grown, vertically aligned carbon nanofibers was measured using a custom-built atomic force microscope placed inside a scanning electron microscope. The internal structure of the nanofiber was best modeled as dual-phase, composed of an inner graphitic core covered with a tapered amorphous carbon shell. It was found that the fibers have a relatively low bending stiffness, with Young's modulus values of about $10 \mathrm{GPa}$ for the inner core and $65 \mathrm{GPa}$ for the outer shell. The low Young's modulus of the inner core is attributed to a non-zero angle between the graphitic sheets and the nanofiber axis. The weak shear modulus between graphitic sheets thereby dominates the mechanical behaviour of the fibers. (C) 2013 AIP Publishing LLC. [http://dx.doi.org/10.1063/1.4803853]
\end{abstract}

\section{INTRODUCTION}

Vertically aligned carbon nanofibers (VACNFs) are synthesized in a plasma-enhanced chemical vapor deposition (PECVD) process in which the interaction between the electrostatic field present in the plasma and the catalyst particles at the tip of the nanofibers results in the vertical alignment. ${ }^{1}$ Since the synthesis is catalytic, one can control the position and the diameter of individual VACNFs by patterning the catalyst, and control their length through the growth time. As a result of their synthesis process and their resemblance to carbon nanotubes (CNTs), VACNFs have been proposed to be used in a number of applications, where some have already been demonstrated including electron emitters, ${ }^{2}$ gene delivery arrays, ${ }^{3}$ and nanoelectromechanical systems. ${ }^{4-7}$

CNTs are similar to VACNFs and their mechanical properties have been thoroughly studied. The Young's modulus, $E$, for tubes with a high crystallinity has been found to be about $1 \mathrm{TPa}^{8}{ }^{8}$ This is similar to the in-plane elastic constant of graphite $c_{11}=1.06 \mathrm{TPa},{ }^{9}$ not surprisingly since CNTs consist of concentric cylinders of graphene. Misalignment of the graphitic planes, along with defects, can however drastically reduce the Young's modulus by almost two orders of magnitude. ${ }^{8}$

VACNFs consist of cone- or cup-shaped graphitic layers stacked in one another, ${ }^{10}$ and the graphitic planes form an angle $\theta$ with the tube axis, see Fig. 1. Because of this angle, the graphitic cups can shear against each other when a load is applied to the fiber. The shear modulus of graphite is very low, $c_{44}<5 \mathrm{GPa},{ }^{11}$ and it dominates the mechanical behaviour of graphite when loads are applied non-parallel to the graphite planes. ${ }^{8}$ One would, therefore, expect that shear will dominate the mechanical behaviour of VACNFs as well. A molecular dynamics study found a negligible load transfer between the cones, resulting in an axial Young's modulus of

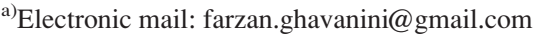

about $30 \mathrm{GPa} .{ }^{12}$ This value was obtained for a CNF consisting of 4 cones having $\theta=30^{\circ}$ and the authors predicted this value to be even lower for a higher number of cones, as in real VACNFs. There have been a few attempts to measure the Young's modulus of VACNFs thus far. In Table I, we summarize these studies along with studies of similar materials. As can be seen in Table I, there is a large spread in the reported values for $E$, and the bending stiffness of individual VACNFs have not been characterized before.

In the present work, the bending stiffness of as-grown individual VACNFs was directly measured inside a scanning electron microscope (SEM) using a custom made atomic force microscopy (AFM) instrument. ${ }^{13}$ Individual VACNFs were pushed sideways against the AFM cantilever as shown in Figs. 2(a) and 2(b), and their spring constants were obtained from the bending measurement data. We find that VACNFs must be modelled as a dual-phase material composed of a graphitic inner core encapsulated within an amorphous carbon shell. By fitting the measurement data to the developed model, we derive Young's moduli of $10 \mathrm{GPa}$ and $65 \mathrm{GPa}$ corresponding to the inner core and the outer shell, respectively.

\section{EXPERIMENTAL SETUP AND SAMPLE PREPARATION}

The custom-made AFM instrument was equipped with a piezoresistive force sensor incorporated into the cantilever ${ }^{17}$ and was controlled by software and electronics from Nanofactory Instruments. ${ }^{18}$ Samples were mounted on a positioning system where both coarse and fine motion are performed using a single tube-scanner. ${ }^{19}$ A schematic of the instrument is shown in Fig. 2(c). The motion of the tubescanner was calibrated in the SEM by taking images at several piezo voltages covering the distance range needed for the force measurements. The output voltage of the piezoresistive sensor, i.e., its sensitivity, versus cantilever deflection was calibrated by pushing a hard material (silicon surface) 

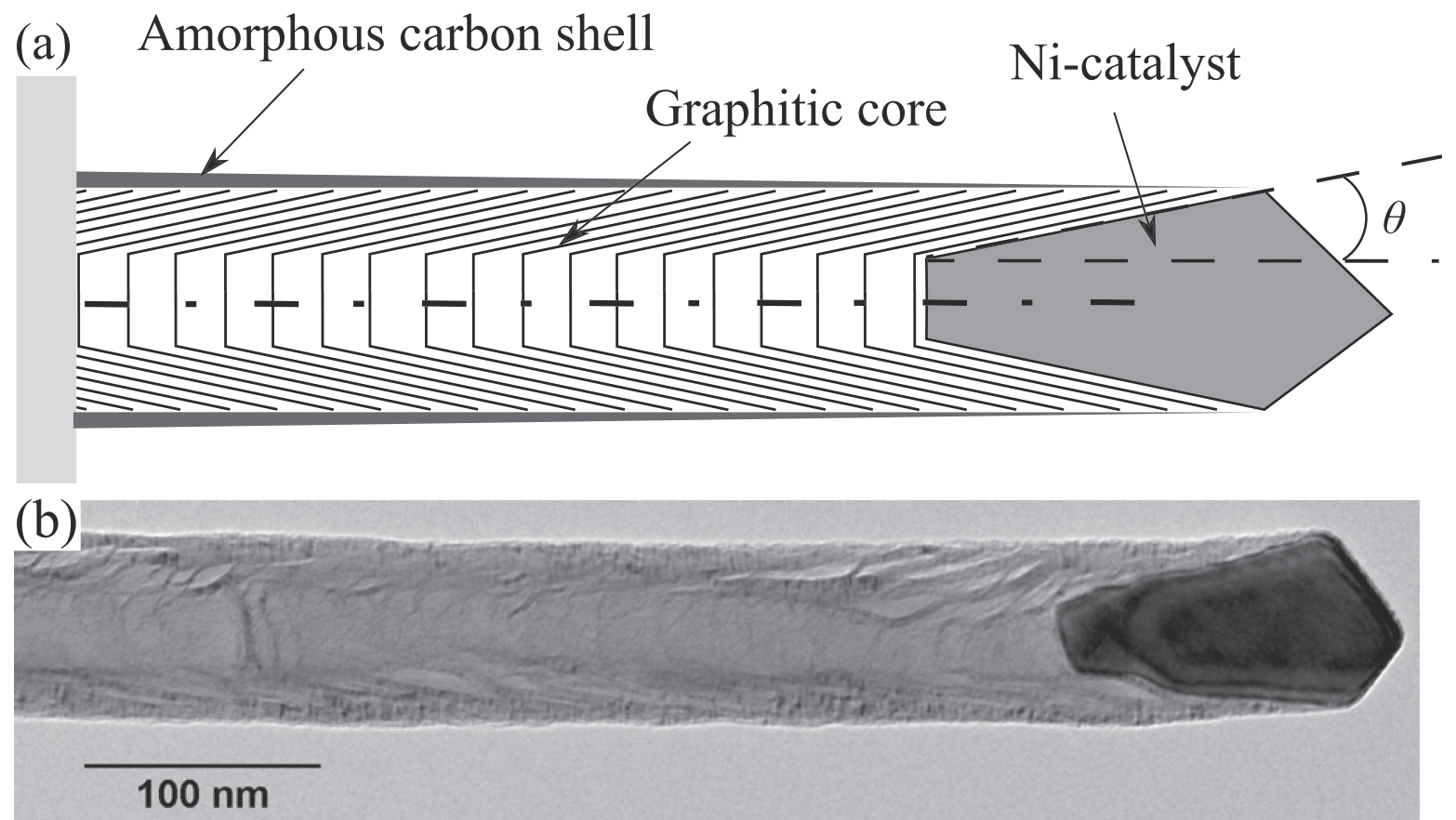

FIG. 1. (a) Schematic drawing of the VACNF structure. Amorphous carbon is deposited onto the graphitic core continuously during growth, thus rendering a tapered structure. (b) TEM image of a VACNF.

against it. The spring constant of the cantilever was obtained by pushing it against a commercially available pre-calibrated cantilever. ${ }^{20}$ The AFM instrument was mounted inside a LEO 1530 FEG-SEM, operated at an acceleration voltage of $8 \mathrm{kV}$. In order to minimize electron-beam induced deposition of amorphous carbon when imaging with SEM, the AFM instrument along with the sample was loaded inside the SEM chamber at least $10 \mathrm{~h}$ before the experiment, giving a pressure of about $5 \times 10^{-7}$ mbar during measurements. Furthermore, the samples were only exposed to the electron beam for the initial positioning and the electron beam was kept deflected away from them during the force measurements.

VACNFs were grown in a PECVD process using a 2-in. AIXTRON Black Magic reactor in a $\mathrm{C}_{2} \mathrm{H}_{2} / \mathrm{NH}_{3}$ atmosphere from $12 \mathrm{~nm}$-thick Ni catalyst seeds deposited on top of reactively sputtered TiN substrates. Electron-beam lithography was adopted to pattern the catalyst layer into circular seeds in the diameter range of $40 \mathrm{~nm}$ to $200 \mathrm{~nm}$. The samples were divided into two different groups. In both groups, the synthesis process started by $1 \mathrm{~h}$ annealing at $580^{\circ} \mathrm{C}$ in nitrogen at 6 mbar. Then, in one group (group A), the growth was carried

TABLE I. Studies on the mechanical properties of VACNFs.

\begin{tabular}{lc}
\hline \hline Material and method & $E[\mathrm{GPa}]$ \\
\hline Nanoindentation on VACNF forests (Ref. 14). & $900-1230$ \\
Electromechanical resonance on a single & \\
cantilevered CNF (Ref. 15) & 410 \\
Nanoindentation on a single as-grown VACNF (Ref. 16) & 816 \\
Molecular dynamics on a CNF having four & \\
shells with $\theta=30^{\circ}$ (Ref. 12) & 30 \\
Modeled as a one-phase material [This study] & $10-120$ \\
Modeled as a dual-phase material [This study] & Core: 10 Shell: 65 \\
\hline
\end{tabular}

out at a plasma power of $40 \mathrm{~W}$, a $\mathrm{C}_{2} \mathrm{H}_{2} / \mathrm{NH}_{3}$ gas ratio of $1 / 4$, a chamber pressure of $3.5 \mathrm{mbar}$, and substrate temperature of $635^{\circ} \mathrm{C}$. This set of growth parameters allowed for some amount of amorphous carbon deposits on the VACNFs' sidewalls as seen in Fig. 3(a). In group B, the growth conditions were tuned ${ }^{21}$ in order to minimize the sidewall deposits by increasing the chamber pressure to 8.9 mbar and reducing the $\mathrm{C}_{2} \mathrm{H}_{2} / \mathrm{NH}_{3}$ gas ratio to $1 / 6$. As a result, the VACNFs in group A were considerably more tapered than those belonging to group B (see Fig. 3(b)).

The internal structure of the VACNFs was studied using a JEOL (JEM 2100) TEM equipped with a $\mathrm{LaB}_{6}$ cathode and a digital camera from Gatan (SC1000 Orius). All images were acquired using an acceleration voltage of $100 \mathrm{kV}$.

The dimensions of the VACNFs were measured from high magnification SEM images taken after the force measurements to avoid beam induced modifications to the fibers. Special care was taken when measuring the VACNFs diameter since the accuracy of the calculated stiffness strongly depends on it. ${ }^{13}$ In order to quantify the taperedness of the VACNFs, we define a taperedness factor, $\alpha$, as the following:

$$
\alpha \equiv \frac{r_{b}-r_{t}}{l}
$$

where $r_{b}, r_{t}$, and $l$ are the base radius, the tip radius, and the length of a VACNF, respectively. When modelling the fibers, the values of $r_{t}$ and $l$ were measured at the point where the force was applied to reflect the effective length.

\section{RESULTS AND DISCUSSIONS}

In situ bending measurements were performed on five VACNFs from Group A and five VACNFs from Group B. 


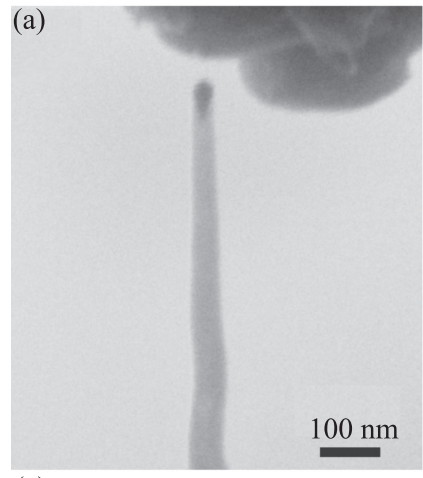

(c)
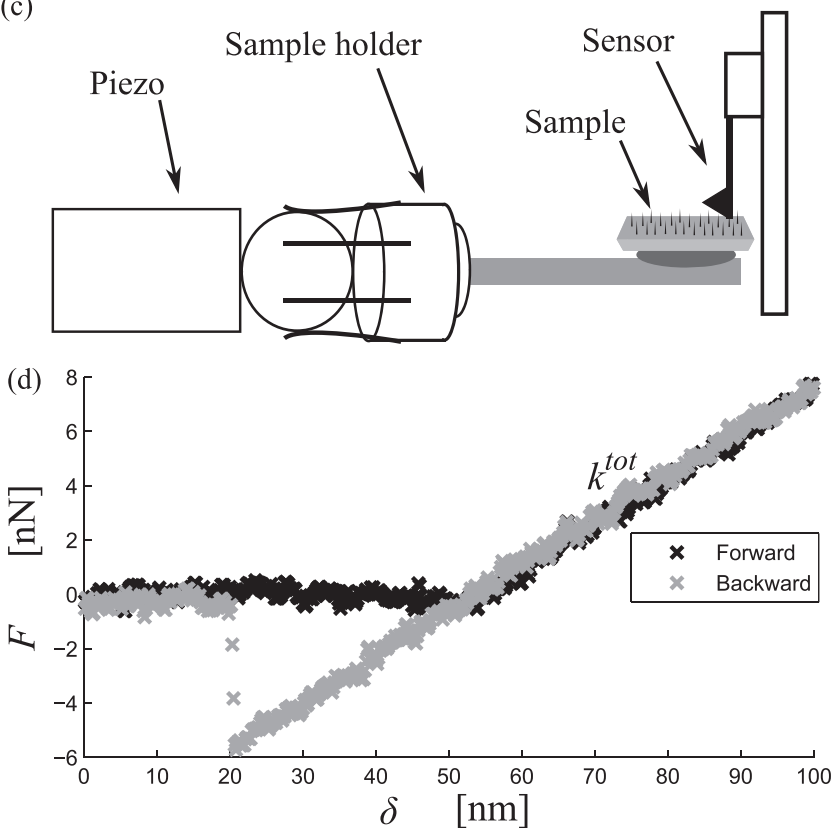

FIG. 2. (a) and (b) SEM images showing the in situ deflection of a VACNF (a) without a load and (b) during bending. (c) Schematic of the instrument. (d) Typical force vs. deflection curve obtained for forward and backward motion.

By knowing the spring constant of the cantilever, $k_{c}$, those of the VACNFs, $k_{v}$, were calculated using the spring constant $k_{\text {tot }}$ obtained from the $F-\delta$ curves (see Fig. 2(d)). In a simple approach, one can assume a uniform material with a single Young's modulus for the VACNFs as shown in Fig. 4(a). In
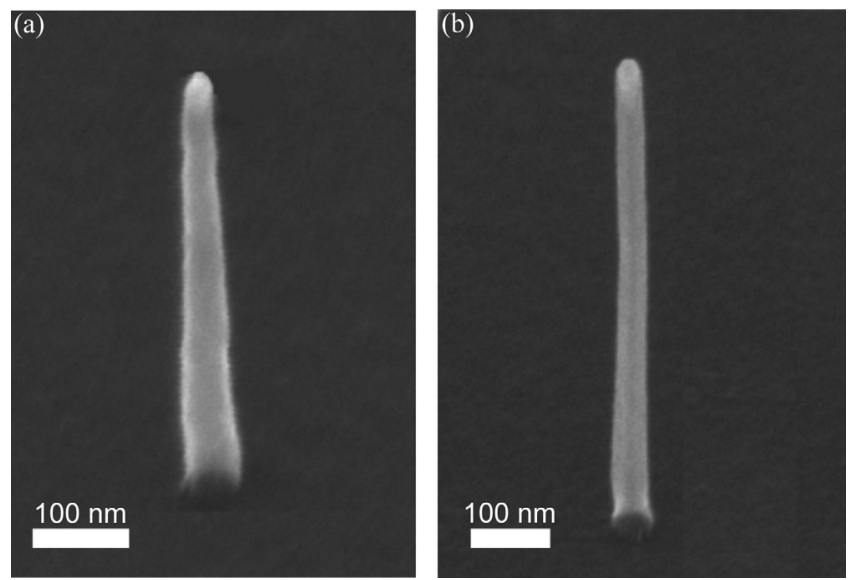

FIG. 3. Representative SEM images of each synthesis group, where (a) Group A, are more tapered than (b) Group B. (a)

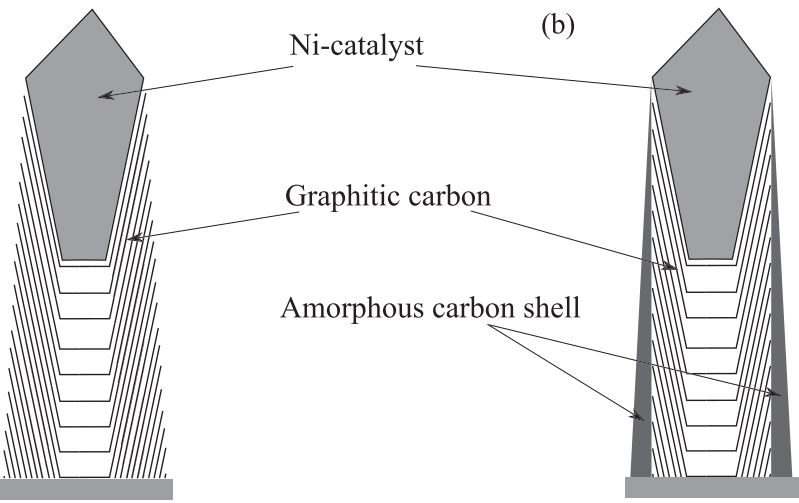

FIG. 4. Schematic representation of two different modeling approaches of the VACNFs. (a) Single-phase model with a tapered uniform material. (b) Dual-phase model assigns two different Young's moduli, one to a constant diameter inner core and another to a tapered outer shell.

this way, the spring constant of a VACNF, i.e., the ratio between the applied force and the resulting deflection at its tip is correlated to its Young's modulus, $E$, by ${ }^{22}$

$$
k_{v}=\frac{3 \pi E r_{t} r_{b}^{3}}{4 l^{3}} .
$$

The obtained values of Young's modulus are plotted as a function of the fiber taperedness factor in Fig. 5. The error bars in Fig. 5 originate from the uncertainty in measuring the VACNFs dimensions and the calibration inaccuracy of the cantilever's spring constant. Clearly, the more conical VACNFs of group A have larger Young's moduli, than those of group B. Moreover, it seems that an increase in the Young's modulus within each group is correlated with an increase in the taperedness factor, i.e., with an increase in the amorphous deposits on the VACNFs sidewalls. This observation suggests that the amorphous carbon shell is stiffer than the graphitic inner core, and a better model is required. Therefore, we developed a dual-phase model in which the VACNFs are composed of an inner graphitic rod inside a conical shell of amorphous carbon as illustrated in Fig. 4(b).

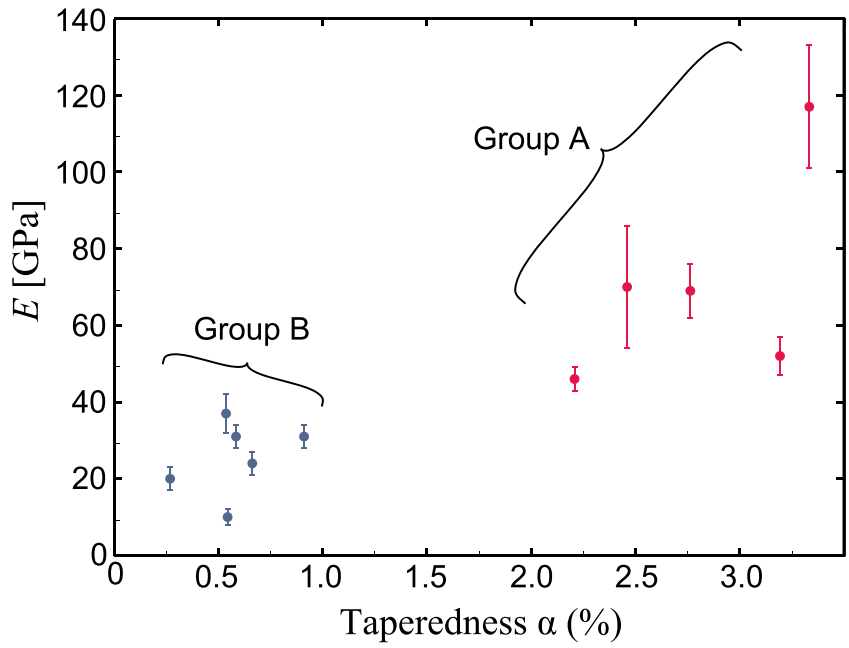

FIG. 5. Young's modulus of the VACNFs versus their taperedness factor as estimated using the single-phase model. 


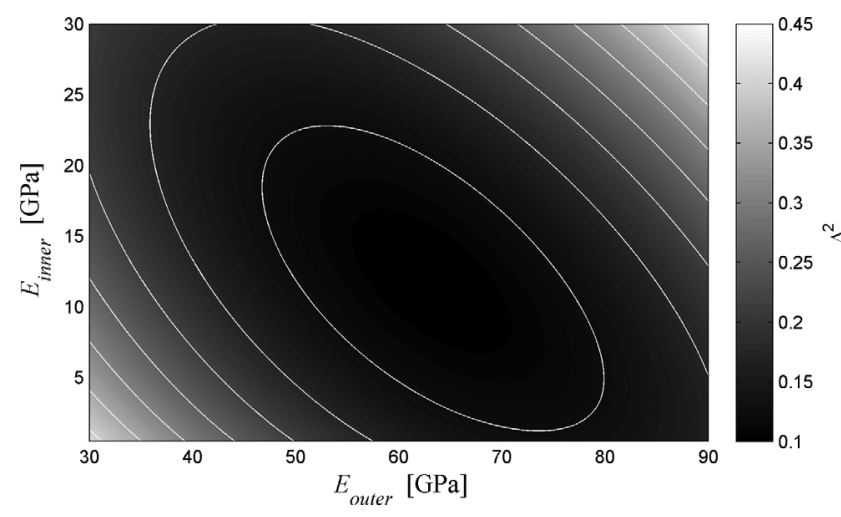

FIG. 6. The obtained variance as a function of $E_{\text {inner }}$ and $E_{\text {outer }}$ in the dualphase model.

Such a structure has been suggested previously based on TEM studies. ${ }^{23}$ From our TEM images, we find a similar structure of our fibers, although there are also many defects.

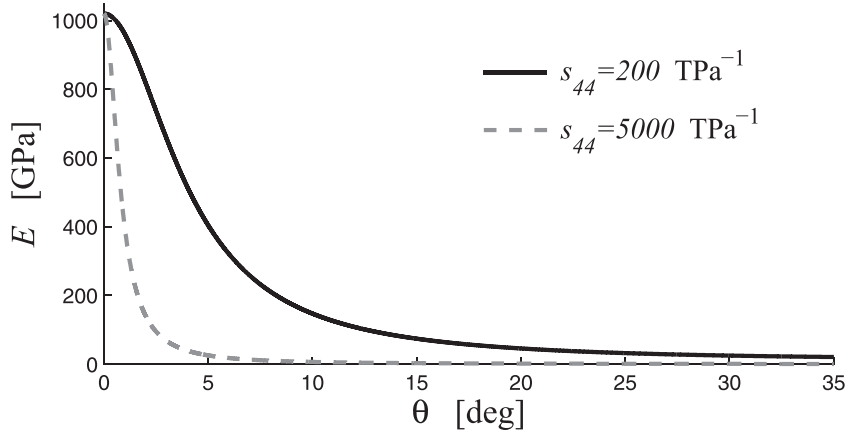

FIG. 7. Dependence of the elastic modulus on the angle between the graphitic sheets and the tube axis in a single graphite crystal, using Eq. (5) with the two extreme values of $s_{44}$.

In the dual-phase model, we assign a different Young's modulus to each phase and determine the stiffness of the resulting structure using 22

$$
k_{v}=\frac{\pi \alpha^{3} \beta^{3} E_{\text {outer }} r_{t}}{2\left(\beta^{2}-1\right) \arctan \left(\frac{\beta\left(r_{b}-r_{t}\right)}{\beta^{2} r_{t}+r_{b}}\right)+\left(\beta^{2}+1\right) \ln \left(\frac{(1+\beta)\left(r_{b}-\beta r_{t}\right)}{(1-\beta)\left(r_{b}+\beta r_{t}\right)}\right)+2 \beta \ln \left(\frac{\left(1-\beta^{2}\right)\left(r_{b}^{2}+\beta^{2} r_{t}^{2}\right)}{\left(1+\beta^{2}\right)\left(r_{b}^{2}-\beta^{2} r_{t}^{2}\right)}\right)},
$$

where $E_{\text {outer }}$ and $E_{\text {inner }}$ stand for the Young's modulus of the outer shell and the inner rod, respectively, and $\beta$ is given by

$$
\beta=\frac{E_{\text {outer }}-E_{\text {inner }}}{E_{\text {outer }}} \text {. }
$$

Equation (3) can be used to find the best fit to the measured spring constants, by plotting the variance, $\Delta^{2}$, for different values of $E_{\text {outer }}$ and $E_{\text {inner }}$. Fig. 6 visualizes this, and a minimum for $\Delta^{2}$ is obtained for $E_{\text {inner }} \approx 10 \mathrm{GPa}$ and $E_{\text {outer }} \approx$ $65 \mathrm{GPa}$.

The relatively low stiffness of the inner graphitic core can be attributed to the non-zero angle between the constituent graphitic sheets and the VACNF's vertical axis. The stiffness of the inner part is thereby dominated by the shear modulus between the graphitic layers, which is about $5 \mathrm{GPa}$ for highly crystalline graphite, but can be as low as $1 \mathrm{GPa}$ when glissile basal-plane dislocations are present. ${ }^{11}$ The cup-stacked structure has a lower density near the fiber axis that covers less than $50 \%$ of the inner phase radius. Such a region on the fiber axis has a negligible contribution to the Young's modulus, which allows us to treat the inner phase as homogeneous. ${ }^{20}$ If the inner phase of the VACNFs is approximated by a single graphite crystal in which the graphitic planes form an angle $\theta$ with respect to the tube axis, then the elastic modulus is given by the following equation: ${ }^{8}$

$$
\frac{1}{E}=s_{11}\left(1-\gamma^{2}\right)^{2}+s_{33} \gamma^{4}+\left(2 s_{13}+s_{44}\right) \gamma^{2}\left(1-\gamma^{2}\right),
$$

where $\gamma=\sin (\theta)$ and $s_{i j}$ are the elastic compliances of bulk graphite having values: ${ }^{9} s_{11}=0.98 \mathrm{TPa}^{-1}, s_{33}=27.5 \mathrm{TPa}^{-1}$, and $s_{13}=-0.33 \mathrm{TPa}^{-1}$. For $s_{44}=1 / c_{44}$, the reported values differ and are in the range $200 \leq s_{44} \leq 5000 \mathrm{TPa}^{-1} \cdot{ }^{11}$ Equation (5) describes a considerable drop in the elastic modulus as the angle $\theta$ increases (see Fig. 7).

From TEM-images, we found that the angle between the graphitic planes and the fiber's axis was in the range $10^{\circ}<\theta<30^{\circ}$. From Eq. (5) and Fig. 7, $E$ is in the range $7<E<150 \mathrm{GPa}$ for an angle of $\theta=10^{\circ}$, whereas it drops rapidly to the range $1<E<25 \mathrm{GPa}$ when the angle is increased to $30^{\circ}$. The strong dependence of the elastic modulus on the angle of the graphitic sheets and the defect density may also explain the relatively large fitting error that we observe in Fig. 6. In other words, even small disparities in the internal structure of the VACNFs, as expected from those grown in a PECVD process, could translate into considerable variations of the Young's modulus.

As can be seen in Fig. 6, we obtain a Young's modulus of $65 \mathrm{GPa}$ for the outer amorphous shell. This is similar to the reported elastic modulus of amorphous carbon films which is in the range of $100 \mathrm{GPa}$ to $500 \mathrm{GPa}^{24}$

\section{CONCLUSIONS}

We presented a method in which the bending stiffness of as-grown individual VACNFs was directly measured inside a SEM using a custom made AFM instrument. It was shown that the previous assumption of a uniform internal structure for VACNFs is inadequate for describing their mechanical properties and that a dual-phase model composed of a graphitic core inside an amorphous carbon shell provides a better description. We derived two Young's moduli of 
$10 \mathrm{GPa}$ and $65 \mathrm{GPa}$ corresponding to the inner core and the outer shell, respectively. The relative weakness of the inner core is attributed to the non-zero angle between the constituent graphitic sheets and the VACNFs' vertical axis which renders the shear modulus between the sheets the dominant factor in the mechanical behavior of the nanofibers. The disparity between the Young's moduli of the inner core and the outer shell introduces an opportunity to tailor the mechanical stiffness of VACNFs for a specific application by controlling the amount of amorphous carbon deposits on their sidewalls. This can be done by tuning the process parameters during the VACNFs synthesis.

\section{ACKNOWLEDGMENTS}

Financial support from the Swedish research council (Project No. 2010-4324) and Swedish foundation for strategic research (Project No. RE07-0004) are gratefully acknowledged.

${ }^{1}$ V. I. Merkulov, A. V. Melechko, M. A. Guillorn, D. H. Lowndes, and M. L. Simpson, Appl. Phys. Lett. 79, 2970 (2001).

${ }^{2}$ M. A. Guillorn, A. V. Melechko, V. I. Merkulov, E. D. Ellis, C. L. Britton, M. L. Simpson, D. H. Lowndes, and L. R. Baylor, Appl. Phys. Lett. 79, 3506 (2001).

${ }^{3}$ D. G. J. Mann, T. E. Mcknight, A. V. Melechko, M. L. Simpson, and G. S. Sayler, Biotechnol. Bioeng. 97, 680 (2007).

${ }^{4}$ J. E. Jang, S. N. Cha, Y. J. Choi, D. J. Kang, T. P. Butler, D. G. Hasko, J. E. Jung, J. M. Kim, and G. A. J. Amaratunga, Nat. Nanotechnol. 3, 26 (2008).

${ }^{5}$ J. E. Jang, S. N. Cha, Y. Choi, G. A. J. Amaratunga, D. J. Kang, D. G. Hasko, J. E. Jung, and J. M. Kim, Appl. Phys. Lett. 87, 163114 (2005).
${ }^{6}$ F. A. Ghavanini, P. Enoksson, S. Bengtsson, and P. Lundgren, J. Appl. Phys. 110, 021101 (2011).

${ }^{7}$ O. Y. Loh and H. D. Espinosa, Nat. Nanotechnol. 7, 283 (2012).

${ }^{8}$ J.-P. Salvetat, A. J. Kulik, J.-M. Bonard, G. A. D. Briggs, T. Stöckli, K. Méténier, S. Bonnamy, F. Béguin, N. A. Burnham, and L. Forró, Adv. Mater. 11, 161 (1999).

${ }^{9}$ O. L. Blakslee, D. G. Proctor, E. J. Seldin, G. B. Spence, and T. Weng, J. Appl. Phys. 41, 3373 (1970).

${ }^{10}$ A. V. Melechko, V. I. Merkulov, T. E. McKnight, M. A. Guillorn, K. L. Klein, D. H. Lowndes, and M. L. Simpson, J. Appl. Phys. 97, 041301 (2005).

${ }^{11}$ M. Grimsditch, J. Phys. C Solid State 16, 143 (1983).

${ }^{12}$ C. Wei and D. Srivastava, Appl. Phys. Lett. 85, 2208 (2004).

${ }^{13}$ H. Jackman, Lic. thesis, Karlstad University, Karlstad, 2012.

${ }^{14}$ H. Qi, K. Teo, K. Lau, M. Boyce, W. Milne, J. Robertson, and K. Gleason, J. Mech. Phys. Solids 51, 2213 (2003).

${ }^{15}$ A. Eriksson, S. Lee, A. A. Sourab, A. Isacsson, R. Kaunisto, J. M. Kinaret, and E. E. B. Campbell, Nano Lett. 8, 1224 (2008).

${ }^{16}$ A. B. Kaul, K. G. Megerian, A. T. Jennings, and J. R. Greer, Nanotechnology 21, 315501 (2010).

${ }^{17}$ A. Nafari, D. Karlen, C. Rusu, K. Svensson, H. Olin, and P. Enoksson, J. Microelectromech. Syst. 17, 328 (2008).

${ }^{18}$ See www.nanofactory.com for more information.

${ }^{19}$ K. Svensson, Y. Jompol, H. Olin, and E. Olsson, Rev. Sci. Instrum. 74, 4945 (2003).

${ }^{20}$ H. Jackman, P. Krakhmalev, and K. Svensson, Appl. Phys. Lett. 98, 183104 (2011).

${ }^{21}$ F. A. Ghavanini, M. Lopez-Damian, D. Rafieian, K. Svensson, P. Lundgren, and P. Enoksson, Sens. Actuators, A 172, 347 (2011).

${ }^{22}$ See supplementary material at http://dx.doi.org/10.1063/1.4803853 for a derivation.

${ }^{23}$ V. I. Merkulov, M. A. Guillorn, D. H. Lowndes, M. L. Simpson, and E. Voelkl, Appl. Phys. Lett. 79, 1178 (2001).

${ }^{24}$ B. Schultrich, H.-J. Scheibe, G. Grandremy, and D. Schneider, Phys. Status Solidi A 145, 385 (1994). 